\title{
Detecting Outliers using R Package in Fitting Data with Linear and Nonlinear Regression Models
}

\author{
Manimannan G ${ }^{1 *}$, M. Salomi ${ }^{2}$, R. Lakshmi Priya ${ }^{3}$, Saranraj R. ${ }^{4}$ \\ ${ }^{I}$ Assistant Professor, Department of Statistics, TMG College of Arts and Science, Chennai. \\ ${ }^{2}$ Assistant Professor, Department of Statistics, Madras Christian College, Chennai \\ ${ }^{3}$ Assistant Professor, Department of Statistics, Dr. Ambedkar Government Arts College, Vyasarpadi, Chennai. \\ ${ }^{4}$ Assistant Professor, Department of Statistics, St. Joseph's College of Arts and Science, Cuddolore
}

*Corresponding Author: Manimannan G, Assistant Professor, Department of Statistics, TMG College of Arts and Science, Chennai.

\begin{abstract}
:
Background

Linear and non linear regression analysis assumes scatter of data, fitting of straight line or normal distribution. An outlier is an extreme observation when the residual is larger in absolute value when compared with other observed data set. The detection of outlier can be defined as the process of detecting and subsequently excluding outliers from the given set of data. Outlier can dominate sum of square calculation and lead to misleading results. In this paper, an attempt is made to detect the outlier of linear and non linear regression models using new approach of standardized scores of detecting outliers without the use of predicted values.

Results

First describe the methods of linear and non linear regression model. The data fitted four times of regression model. Initially the original data to fit linear regression model and deduct outlier and visualize the results. Second method is to fit linear regression without outlier and visualize the data. Third method is to fit non linear regression with original data and visualize the results. Last method is to fit non linear regression for removal of outlier data and visualize the results. In both the methods only one outlier is identified and removed using standardized score. The primary data sources were collected from case sheets in a private hospital at Bangalore. In this analysis, two parameters are Age and SBP (Systolic Blood Pressure) were used. Blood pressure is measured in two types, top level Systolic blood pressure and Bottom level DBP (Diastolic Blood Pressure). Both are measured from arteries during the contraction of heart muscles of the patient.

Conclusion

The linear and nonlinear regression model fitted for original and outlier removed data. The result of linear and non linear regression for the original data is an average model. In both the models, $R^{2}$ value is less than 0.5. After removal of outlier better fit of linear and nonlinear regression model is achieved. The $R^{2}$ values are more than 0.7. The $F$ and t statistic are significant in two models. The scatter plot clearly visualized the outlier and without outlier data for different plots. The summary statistics of both regression models results are expressed in following section. A new approach for detecting outliers without the use of predicted values have been proposed which is quite useful in detecting outliers that detects the outliers as similar to residual and standardized residual method.

Keywords: Outlier, Linear Regression, Nonlinear Regression, Summary statistics, Residual, Predicted, Standardized Analysis and Scatter Plot Visualization.
\end{abstract}

\section{INTRODUCTION}

Regression analysis is one of the most widely used statistical tools for analyzing multifactor database. It is appealing because it provides a conceptually simple method for investigating functional relationships among variables. Regression analysis is concerned with the study of dependence of one variable, on one or more other variables, called the explanatory variables, with a view to estimating and or predicting the mean or average value of the former in terms of the known or fixed in values of the latter. The problem of model selection in linear regression model has received much attention in statistical literature. For a detailed study, refer to Draper and Smith, 1981 [1]. The statistical tools available for analysis of data are "regression." Theory of regression deals with prediction of one or more variables, called "dependent (response) variables" on the basis of other variables called "independent variables." The dependent variable is also called "criterion variable." For independent 
variable names such as "predictor" or "explanatory" variables are also common. When a model is used to explain dependent variable in terms of independent variable it assumes a linear relationship between them and arrive at a linear regression model or otherwise a non-linear regression model. Framstad et.al, 1985 [2] suggest that in simple linear regression model, the difference method can be used for detecting outliers

\section{Database}

The primary data sources were collected from the case sheets in private hospital at Bangalore. The database had many parameters like DBP, BMI, Height, Weight, Hb, RBC, ESR, etc (Manimannan G. et.al, 2020 [3]. In this research paper, the researcher concentrates only on Age and SBP of the respondents. In this analysis two parameters namely, Age and SBP (Systolic Blood Pressure) were used. Blood pressure is measured in two types namely, top level Systolic blood pressure (SBP) and Bottom level DBP (Diastolic Blood Pressure). Both are measured from arteries during the contraction of heart muscles of the patient.

\section{MODEL DESCRIPTION}

\subsection{Linear Regression}

The equation of a straight line functional relationship of $Y$ on $X$ is given by Montgomery, et al .[4].

$Y=\beta_{0}+\beta_{1} X$

which is known as simple linear regression of $Y$ on $X . \beta_{0}$ and $\beta_{1}$ are called parameters, and should be found in equation (1) means that for a given $X_{i}$, a corresponding $Y_{i}$ consists of $Y=\beta_{0}+\beta_{1} X_{i}$ and an $\varepsilon_{i}$ by which an observation may fall off the true regression line. On the basis of the information available from the observations $\beta_{0}$ and $\beta_{1}$ has to be estimated.

This model is given by

$Y=\beta_{0}+\beta_{1} X+\varepsilon$

which is called simple linear regression model. The term $\varepsilon$ is a random variable and is called "error term". Finding $\beta_{0}$ and $\beta_{1}$ from $\left(X_{i}, Y_{i}\right) i=1,2, \ldots, n$ is called estimation of the parameters. The Ordinary Least Square (OLS) methods can be used to fit the model and estimate the parameter values. The method of least square is used to estimate the parameters $\beta_{0}$ and $\beta_{1}$ that the sum of square of the difference between the observation $Y$ the straight line is minimum from the equation (2).The least square estimators of $\beta_{0}$ and $\beta_{1}$ must satisfy $\hat{\beta}_{0}$ and $\hat{\beta}_{1}$.

The least square estimator of the intercept

$\hat{\beta}_{0}=\underline{y}-\hat{\beta}_{1} \underline{x}$

The least square estimation of the slope $\beta_{1}$

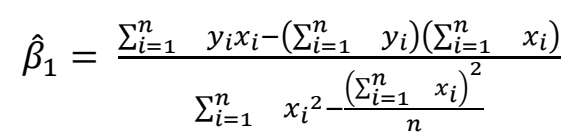

By fitting simple linear regression model is given by

$\hat{Y}=\hat{\beta}_{0}+\hat{\beta}_{1} x$

Equation (5) gives a point estimate of mean of $Y$ for a particular $x$. Equation (4) is the corrected sum of square of $x_{i}$. The difference between the observed value $y_{i}$ and the corresponding fitted value $\hat{Y}$ is a residual. By mathematically the residual of $i^{\text {th }}$ is

$e_{i}=y_{i}-\hat{y}_{i}$

$e_{i}=y_{i}-\left(\hat{\beta}_{0}+\hat{\beta}_{1} x_{i}\right), \quad i=1,2, \ldots, n$

is given by Bipin et.al.[5]. The assumption of regression analysis is as follows :

1. The relationship between response y and the regressor's is linear, at least approximately 
2. The ${ }^{\beta_{j}}$ are unknown parameters to be estimated from the data.

3. It is assumed that errors are normally and independently distributed with zero expectation (mean) and constant variance $\sigma^{2}: e_{i} \sim N I D$ (Normally and Independently Distributed) $\left(0, \sigma^{2}\right)$. I.

4. The errors are uncorrelated.

The assumptions 3 and 4 imply the errors and independent random variables. The approximation of the models is the t or F statistics or $\mathrm{R}^{2}$.

\subsection{Non Linear Regression}

Non linear regression assumes that the scatter of data around the ideal curve follows a normal distribution. This assumption leads to familiar goal of regression to minimize the sum of square of vertical points and curve. Single outlier can dominate the sum of squares calculation which leads to misleading results. As suggested by Hample [6, 7] .It follows the following three steps.

1. Fit a curve using a new robust nonlinear regression method.

2. Analyze the residual of the robust fit, and determine whether one or more variable are outliers

3. Remove the outliers, and perform ordinary least square regression on the remaining data.

Outlier values can seriously disturb the least square. In figure 1 an outlier falls from the line suggested by the rest of the data. If this plot is really an outlier then the estimate of the intercept might be incorrect and the residual mean square is blown up estimate of $\sigma^{2}$ [8].

Fitting of a polynomial regression model using the power of a single predictor (X) by the method of linear least square.

$\mathrm{Y}=\beta_{0}+\beta_{1} x_{1}+\beta_{2} x_{2}{ }^{2}+\ldots+\beta_{k} x_{k}^{k}$

The polynomial regression co-efficient $\beta_{1}$ to $\mathrm{k}^{\text {th }}$ for each degree . A second order $\mathrm{k}=2$ is polynomial form. By using polynomial regression model in one variable is called as quadratic model.

$$
\begin{aligned}
& E(y)=\beta_{0}+\beta_{1} x_{1}+\beta_{2} x_{2}{ }^{2} \\
& E(y)=f(x)
\end{aligned}
$$

is a quadratic function using this model approximately tangled nonlinear relationship equation (7) we get that $y=X \beta+\varepsilon, x$ is the explanatory variables $n * p X$-matrix .

\section{RESUlt AND DisCUSSION}

The medical data analysis will be executed in the following proposed algorithms using R Studio.

Step 1: The medical data imported from .xlsx file and attach () in R Studio and assign SBP as a dependent variable and Age as a independent variable, ie. $\left(X_{i}, Y_{i}\right) i=1,2, \ldots, n$

Step 2: Get Summary statistics from the given data.

Step 3: To execute linear regression function with $\mathrm{R}$ syntax and summarize the results

Step 4: Predict the given data and get the SBP predicted values.

Step 5. To get the residuals values for the given data

Step 6: To get the standardized values for the given data.

Step 7. To execute scale function of linear regression for the variable Age and get the

Standardized values for given data. Scale function of each element of those values is obtained by subtracting and dividing by standard deviation

Step 8. Repeat step 7 for SBP and get standardized values for the given data

Step 9: To Visualize the results for linear regression with help of different scatter plot. Manimannan G. et.al. [9] 
The above algorithm execute the results of linear regression of Predicted value, Standardized value, Residuals, Scale function value of Age and SBP values. (In Table 1 to 3, Figure 1). The results table and figures show the extreme in the 14th row is highlighted as outlier. In Visualization part, models are fitted and highlighted in the plots. Residuals vs, Fitted, Normal QQ plot, Scale - location and Residuals vs. Leverage. All the four plots shows that the 14 person as an outlier.

The summary statistics shows that the minimum age as 17 and maximum age is 69 , the minimum SBP is 110 and maximum SBP as 260. The fitted linear regression model is $\mathrm{y}=0.9327 \mathrm{x}+100.39, \mathrm{R}^{2}=$ 0.4898 . In this model $R^{2}$ value is less than 0.5 and this model is average. Subsequently the outlier is removed and executes the algorithm step 1 to step 9. Cook's distance is used in linear regression analysis to find influential outliers in a set of predictor variable. It is a way to identify points that negatively affects the regression model. The plot is a combination of each given data leverage and residual values, the higher leverage and residuals, the higher the Cook's. The F and t statistic are highly significant between two variables.

Table1. Descriptive Statistics $(n=45)$

\begin{tabular}{|l|l|l|}
\hline Variables & Age & SBP \\
\hline Mean & 44.42 & 141.82 \\
\hline Meadian & 45.00 & 142.82 \\
\hline Mode & 36 & 120 \\
\hline Standard Deviation & 15.390 & 20.509 \\
\hline Range & 52 & 110 \\
\hline Minimum & 17 & 110 \\
\hline Maximum & 69 & 220 \\
\hline
\end{tabular}

Table2. Linear Regression Summary output for original data

\begin{tabular}{|l|l|}
\hline Regression Statistics \\
\hline R Square & 0.4898 \\
\hline Multiple R & 0.478 \\
\hline Intercept & 100.390 \\
\hline Slope (Age) & 0.932 \\
\hline
\end{tabular}

\section{R Studio Linear Regression output:}

Residuals:

Min 1Q Median 3Q Max

$\begin{array}{lllll}-22.102 & -5.024 & 0.774 & 2.562 & 75.774\end{array}$

Coefficients:

Estimate Std. Error t value $\operatorname{Pr}(>|\mathrm{t}|)$

(Intercept) $100.3908 \quad 6.816014 .729<2 \mathrm{e}-16 * * *$

Age $\quad 0.9327 \quad 0.1452 \quad 6.4258 .79 \mathrm{e}-08 * * *$

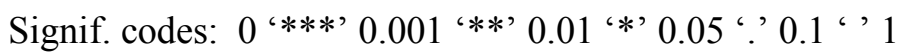

Residual standard error: 14.82 on 43 degrees of freedom

Multiple R-squared: 0.4898， Adjusted R-squared: 0.478

F-statistic: 41.29 on 1 and 43 DF, p-value: 8.791e-08

Table3. Linear Regression of Predicted, Standardized, Residual and nonlinear Standardized and Residual Values

\begin{tabular}{|c|c|c|c|c|c|c|c|}
\hline S.No & $\begin{array}{c}\text { Age } \\
\left(x_{i}\right)\end{array}$ & $\begin{array}{c}\text { SBP } \\
\left(y_{i}\right)\end{array}$ & $\begin{array}{c}\text { Predicted } \\
\text { Value SBP }\end{array}$ & Standardized & Residuals & $\begin{array}{c}\text { Standardized } \\
\left(x_{i}\right)\end{array}$ & $\begin{array}{c}\text { Standardized } \\
\left(y_{i}\right)\end{array}$ \\
\hline 1 & 65 & 162 & 161.0146 & 0.0687 & 0.9854 & 1.3371 & 0.9839 \\
\hline 2 & 46 & 142 & 143.2938 & -0.0883 & -1.2937 & 0.1025 & 0.0087 \\
\hline 3 & 67 & 170 & 162.8799 & 0.4986 & 7.1201 & 1.4671 & 1.3739 \\
\hline 4 & 42 & 124 & 139.5631 & -1.0625 & -15.5630 & -0.1574 & -0.8690 \\
\hline
\end{tabular}


Detecting Outliers using R Package in Fitting Data with Linear and Nonlinear Regression Models

\begin{tabular}{|c|c|c|c|c|c|c|c|}
\hline 5 & 67 & 158 & 162.8799 & -0.3417 & -4.8799 & 1.4671 & 0.7888 \\
\hline 6 & 56 & 154 & 152.6205 & 0.0948 & 1.3795 & 0.7523 & 0.5938 \\
\hline 7 & 64 & 162 & 160.0819 & 0.1334 & 1.9181 & 1.2721 & 0.9839 \\
\hline 8 & 56 & 150 & 152.6205 & -0.1800 & -2.6205 & 0.7523 & 0.3987 \\
\hline 9 & 59 & 140 & 155.4185 & -1.0634 & -15.4180 & 0.9472 & -0.0889 \\
\hline 10 & 34 & 110 & 132.1017 & -1.5165 & -22.1010 & -0.6772 & -1.5517 \\
\hline 11 & 42 & 128 & 139.5631 & -0.7894 & -11.5630 & -0.1574 & -0.6740 \\
\hline 12 & 48 & 130 & 145.1591 & -1.0352 & -15.1590 & 0.2325 & -0.5765 \\
\hline 13 & 39 & 144 & 136.7651 & 0.4945 & 7.2349 & -0.3523 & 0.1062 \\
\hline 14 & 47 & 220 & 144.2264 & 5.1731 & 75.7735 & 0.1675 & 3.8119 \\
\hline 15 & 45 & 138 & 142.3611 & -0.2976 & -4.3611 & 0.0375 & -0.1864 \\
\hline 16 & 47 & 145 & 144.2264 & 0.0528 & 0.7736 & 0.1675 & 0.1549 \\
\hline 17 & 65 & 162 & 161.0146 & 0.0687 & 0.9854 & 1.3371 & 0.9839 \\
\hline 18 & 45 & 135 & 142.3611 & -0.5024 & -7.3611 & 0.0375 & -0.3327 \\
\hline 19 & 17 & 114 & 116.2462 & -0.1593 & -2.2462 & -1.7819 & -1.3566 \\
\hline 20 & 20 & 116 & 119.0442 & -0.2141 & -3.0442 & -1.5869 & -1.2591 \\
\hline 21 & 19 & 124 & 118.1116 & 0.4153 & 5.8884 & -1.6519 & -0.8690 \\
\hline 22 & 36 & 136 & 133.9670 & 0.1392 & 2.0330 & -0.5473 & -0.2839 \\
\hline 23 & 50 & 142 & 147.0245 & -0.3434 & -5.0244 & 0.3624 & 0.0087 \\
\hline 24 & 39 & 120 & 136.7651 & -1.1458 & -16.7650 & -0.3523 & -1.0641 \\
\hline 25 & 21 & 120 & 119.9769 & 0.0016 & 0.0231 & -1.5220 & -1.0641 \\
\hline 26 & 44 & 160 & 141.4284 & 1.2675 & 18.5715 & -0.0274 & 0.8863 \\
\hline 27 & 44 & 160 & 141.4284 & 1.2675 & 18.5715 & -0.0274 & 0.8863 \\
\hline 28 & 53 & 158 & 149.8225 & 0.5601 & 8.1775 & 0.5574 & 0.7888 \\
\hline 29 & 63 & 144 & 159.1492 & -1.0519 & -15.1490 & 1.2072 & 0.1062 \\
\hline 30 & 29 & 130 & 127.4383 & 0.1769 & 2.5617 & -1.0021 & -0.5765 \\
\hline 31 & 25 & 125 & 123.7076 & 0.0899 & 1.2924 & -1.2620 & -0.8203 \\
\hline 32 & 69 & 175 & 164.7453 & 0.7216 & 10.2547 & 1.5970 & 1.6177 \\
\hline 33 & 56 & 154 & 152.6205 & 0.0948 & 1.3795 & 0.7523 & 0.5938 \\
\hline 34 & 64 & 162 & 160.0819 & 0.1334 & 1.9181 & 1.2721 & 0.9839 \\
\hline 35 & 36 & 136 & 133.9670 & 0.1392 & 2.0330 & -0.5473 & -0.2839 \\
\hline 36 & 50 & 142 & 147.0245 & -0.3434 & -5.0244 & 0.3624 & 0.0087 \\
\hline 37 & 39 & 120 & 136.7651 & -1.1458 & -16.7650 & -0.3523 & -1.0641 \\
\hline 38 & 21 & 120 & 119.9769 & 0.0016 & 0.0231 & -1.5220 & -1.0641 \\
\hline 39 & 53 & 158 & 149.8225 & 0.5601 & 8.1775 & 0.5574 & 0.7888 \\
\hline 40 & 63 & 144 & 159.1492 & -1.0519 & -15.1490 & 1.2072 & 0.1062 \\
\hline 41 & 29 & 130 & 127.4383 & 0.1769 & 2.5617 & -1.0021 & -0.5765 \\
\hline 42 & 20 & 116 & 119.0442 & -0.2141 & -3.0442 & -1.5869 & -1.2591 \\
\hline 43 & 19 & 124 & 118.1116 & 0.4153 & 5.8884 & -1.6519 & -0.8690 \\
\hline 44 & 36 & 136 & 133.9670 & 0.1392 & 2.0330 & -0.5473 & -0.2839 \\
\hline 45 & 50 & 142 & 147.0245 & -0.3434 & -5.0244 & 0.3624 & 0.0087 \\
\hline
\end{tabular}

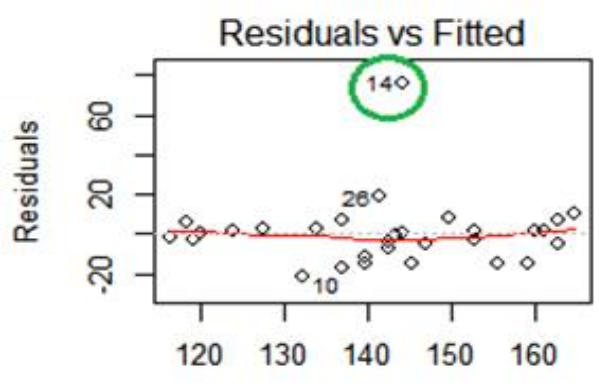

Fitted values

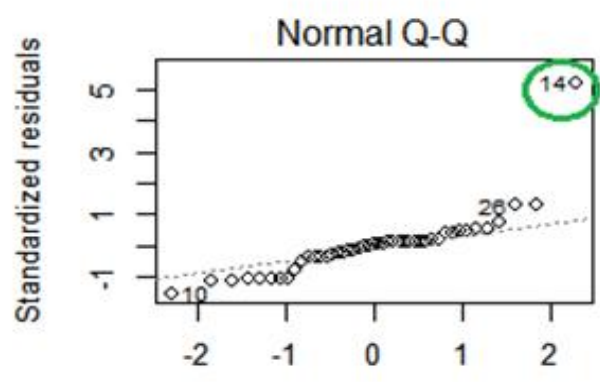

Theoretical Quantiles 


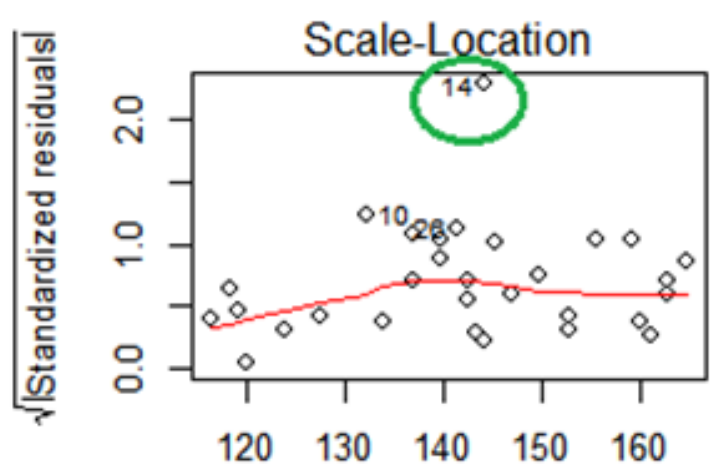

Fitted values

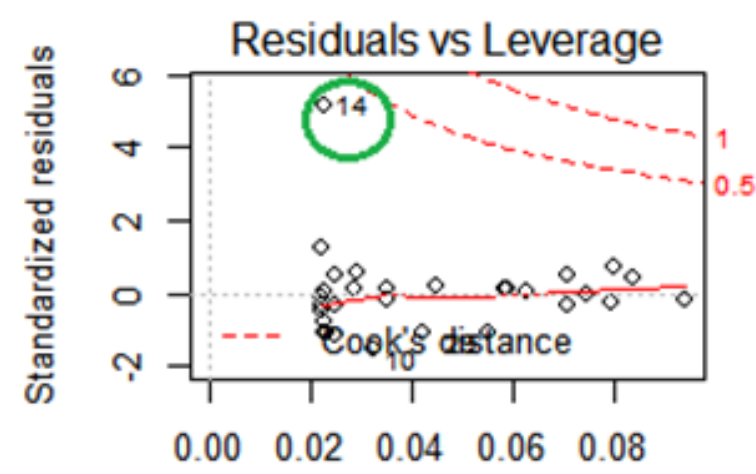

Leverage

Figure1. Scatter plot of Linear Regression models (Original Data).

The previous algorithm again execute the results of linear regression for outlier data and get the Predicted value, Standardized value, Residuals, Scale function value of Age and SBP values. (In Table 4 to 6, Figure 2). The linear regression model improves better. In Visualization parts, models are fitted and highlighted in the plots. They are, Residuals vs, Fitted, Normal QQ plot, Scale - location and Residuals vs. Leverage. All the four plots show better results after removal of outliers.

The summary statistics shows that the minimum age is 17 and maximum age is 69 , the minimum SBP is 110 and maximum SBP as 175 . The fitted linear regression model is $y=0.91359 x+99.51, R^{2}=$ 0.7091. In this model $\mathrm{R}^{2}$ value is closer to .705 and this model is better. Subsequently the non linear regression executes the algorithm step 1 to step 9. Cook's distance is used in linear regression analysis to find influential outliers in a set of predictor variable. It is a way to identify points that negatively affects the regression model. The plot is a combination of each given data leverage and residual values, the higher leverage and residuals, the higher the Cook's. The F and t statistic are highly significant between two variables.

Table4. Descriptive Statistics $n=44$

\begin{tabular}{|l|l|l|}
\hline Variables & Age & SBP \\
\hline Mean & 44.363 & 140.0455 \\
\hline Median & 45 & 141 \\
\hline Mode & 56 & 162 \\
\hline Standard Deviation & 15.562 & 16.88 \\
\hline Range & 52 & 65 \\
\hline Minimum & 17 & 110 \\
\hline Maximum & 69 & 175 \\
\hline
\end{tabular}

Table5. Linear Regression Summary output for original data

\begin{tabular}{|l|l|}
\hline Regression Statistics & \\
\hline R Square & 0.7091 \\
\hline Multiple R & 0.7021 \\
\hline Intercept & 99.519 \\
\hline Slope (Age) & 0.9134 \\
\hline
\end{tabular}

\section{R Studio Linear Regression output (Outlier Removed:}

Residuals:

$$
\begin{array}{ccccc}
\text { Min } & \text { 1Q } & \text { Median } & \text { 3Q } & \text { Max } \\
-20.578 & -3.194 & 1.922 & 3.996 & 20.287
\end{array}
$$

Coefficients:

Estimate Std. Error $\mathrm{t}$ value $\operatorname{Pr}(>|t|)$

(Intercept) $99.519624 .2395123 .47<2 \mathrm{e}-16 * * *$ 
Detecting Outliers using R Package in Fitting Data with Linear and Nonlinear Regression Models

Age $\quad 0.91349 \quad 0.09029 \quad 10.127 .91 \mathrm{e}-13 * * *$

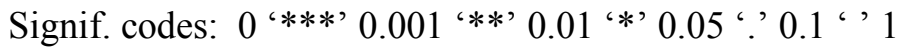

Residual standard error: 9.214 on 42 degrees of freedom

Multiple R-squared: 0.7091， Adjusted R-squared: 0.7021

F-statistic: 102.4 on 1 and 42 DF, p-value: 7.906e-13

Table6. After Removing Outlier from Original Data (Removed $14^{\text {th }}$ row)

\begin{tabular}{|c|c|c|c|c|c|c|c|}
\hline S. No & $\begin{array}{l}\text { Age } \\
\left(x_{i}\right)\end{array}$ & $\begin{array}{l}\text { SBP } \\
\left(y_{i}\right)\end{array}$ & $\begin{array}{l}\text { Predicted } \\
\text { Value SBP }\end{array}$ & Standardized & Residuals & $\begin{array}{c}\text { Standardized } \\
\left(x_{i}\right)\end{array}$ & $\begin{array}{c}\text { Standardized } \\
\left(y_{i}\right)\end{array}$ \\
\hline 1 & 65 & 162 & 158.8966 & 0.3481 & 3.1034 & 1.3260 & 1.3004 \\
\hline 2 & 46 & 142 & 141.5403 & 0.0505 & 0.4597 & 0.1051 & 0.1158 \\
\hline 3 & 67 & 170 & 160.7236 & 1.0451 & 9.2764 & 1.4545 & 1.7743 \\
\hline 4 & 42 & 124 & 137.8863 & -1.5250 & -13.8863 & -0.1519 & -0.9504 \\
\hline 5 & 67 & 158 & 160.7236 & -0.3068 & -2.7236 & 1.4545 & 1.0635 \\
\hline 6 & 56 & 154 & 150.6752 & 0.3675 & 3.3248 & 0.7477 & 0.8266 \\
\hline 7 & 64 & 162 & 157.9831 & 0.4496 & 4.0169 & 1.2618 & 1.3004 \\
\hline 8 & 56 & 150 & 150.6752 & -0.0746 & -0.6752 & 0.7477 & 0.5896 \\
\hline 9 & 59 & 140 & 153.4157 & -1.4886 & -13.4157 & 0.9405 & -0.0027 \\
\hline 10 & 34 & 110 & 130.5784 & -2.2713 & -20.5784 & -0.6659 & -1.7797 \\
\hline 11 & 42 & 128 & 137.8863 & -1.0857 & -9.8863 & -0.1519 & -0.7135 \\
\hline 12 & 48 & 130 & 143.3672 & -1.4685 & -13.3672 & 0.2337 & -0.5950 \\
\hline 13 & 39 & 144 & 135.1458 & 0.9734 & 8.8542 & -0.3447 & 0.2342 \\
\hline 15 & 45 & 138 & 140.6268 & -0.2884 & -2.6268 & 0.0409 & -0.1212 \\
\hline 16 & 47 & 145 & 142.4538 & 0.2796 & 2.5462 & 0.1694 & 0.2935 \\
\hline 17 & 65 & 162 & 158.8966 & 0.3481 & 3.1034 & 1.3260 & 1.3004 \\
\hline 18 & 45 & 135 & 140.6268 & -0.6178 & $\begin{array}{l}-5.6268 \\
\end{array}$ & 0.0409 & -0.2989 \\
\hline 19 & 17 & 114 & 115.0490 & -0.1197 & $\begin{array}{l}-1.0490 \\
\end{array}$ & -1.7583 & -1.5427 \\
\hline 20 & 20 & 116 & 117.7895 & -0.2025 & -1.7895 & -1.5655 & -1.4243 \\
\hline 21 & 19 & 124 & 116.8760 & 0.8081 & 7.1240 & $\begin{array}{l}-1.6298 \\
\end{array}$ & -0.9504 \\
\hline 22 & 36 & 136 & 132.4053 & 0.3960 & 3.5947 & -0.5374 & -0.2396 \\
\hline 23 & 50 & 142 & 145.1942 & -0.3512 & -3.1942 & 0.3622 & 0.1158 \\
\hline 24 & 39 & 120 & 135.1458 & -1.6652 & -15.1458 & -0.3447 & -1.1874 \\
\hline 25 & 21 & 120 & 118.7030 & 0.1464 & 1.2970 & $\begin{array}{l}-1.5013 \\
\end{array}$ & -1.1874 \\
\hline 26 & 44 & 160 & 139.7133 & 2.2272 & 20.2867 & -0.0234 & 1.1820 \\
\hline 27 & 44 & 160 & 139.7133 & 2.2272 & 20.2867 & -0.0234 & 1.1820 \\
\hline 28 & 53 & 158 & 147.9347 & 1.1091 & 10.0653 & 0.5549 & 1.0635 \\
\hline 29 & 63 & 144 & 157.0696 & -1.4600 & -13.0696 & 1.1975 & 0.2342 \\
\hline 30 & 29 & 130 & 126.0109 & 0.4431 & 3.9891 & -0.9872 & -0.5950 \\
\hline 31 & 25 & 125 & 122.3569 & 0.2957 & 2.6431 & -1.2443 & -0.8912 \\
\hline 32 & 69 & 175 & 162.5506 & 1.4095 & 12.4494 & 1.5831 & 2.0705 \\
\hline 33 & 56 & 154 & 150.6752 & 0.3675 & 3.3248 & 0.7477 & 0.8266 \\
\hline 34 & 64 & 162 & 157.9831 & 0.4496 & 4.0169 & 1.2618 & 1.3004 \\
\hline 35 & 36 & 136 & 132.4053 & 0.3960 & 3.5947 & -0.5374 & -0.2396 \\
\hline 36 & 50 & 142 & 145.1942 & -0.3512 & -3.1942 & 0.3622 & 0.1158 \\
\hline 37 & 39 & 120 & 135.1458 & -1.6652 & -15.1458 & -0.3447 & -1.1874 \\
\hline 38 & 21 & 120 & 118.7030 & 0.1464 & 1.2970 & -1.5013 & -1.1874 \\
\hline 39 & 53 & 158 & 147.9347 & 1.1091 & 10.0653 & 0.5549 & 1.0635 \\
\hline 40 & 63 & 144 & 157.0696 & -1.4600 & -13.0696 & 1.1975 & 0.2342 \\
\hline 41 & 29 & 130 & 126.0109 & 0.4431 & 3.9891 & -0.9872 & -0.5950 \\
\hline 42 & 20 & 116 & 117.7895 & -0.2025 & -1.7895 & -1.5655 & -1.4243 \\
\hline 43 & 19 & 124 & 116.8760 & 0.8081 & 7.1240 & $\begin{array}{l}-1.6298 \\
\end{array}$ & -0.9504 \\
\hline 44 & 36 & 136 & 132.4053 & 0.3960 & 3.5947 & -0.5374 & -0.2396 \\
\hline 45 & 50 & 142 & 145.1942 & -0.3512 & $\begin{array}{l}-3.1942 \\
\end{array}$ & 0.3622 & 0.1158 \\
\hline
\end{tabular}




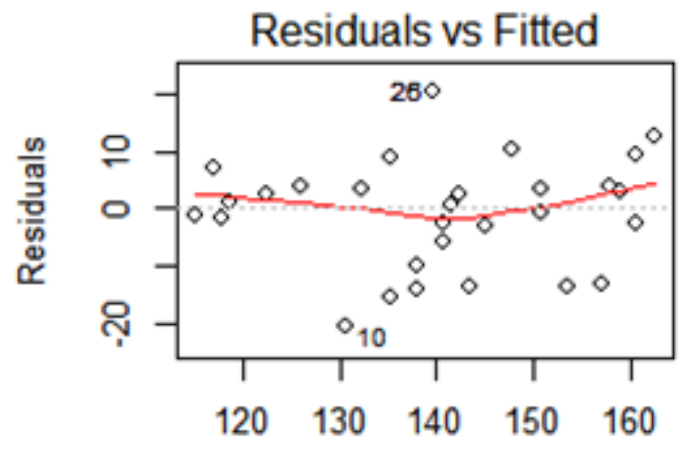

Fitted values

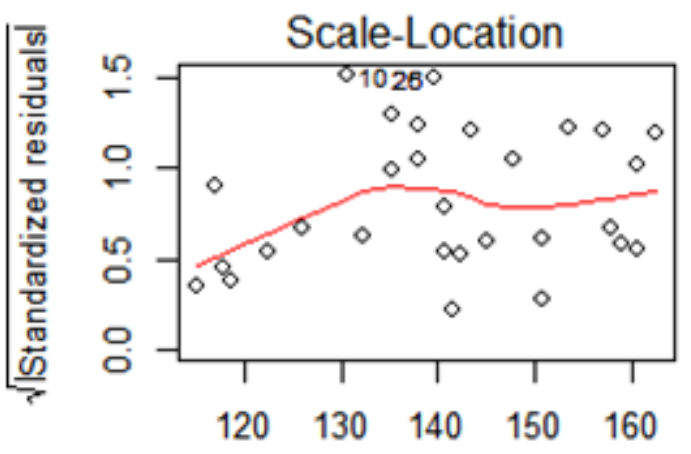

Fitted values

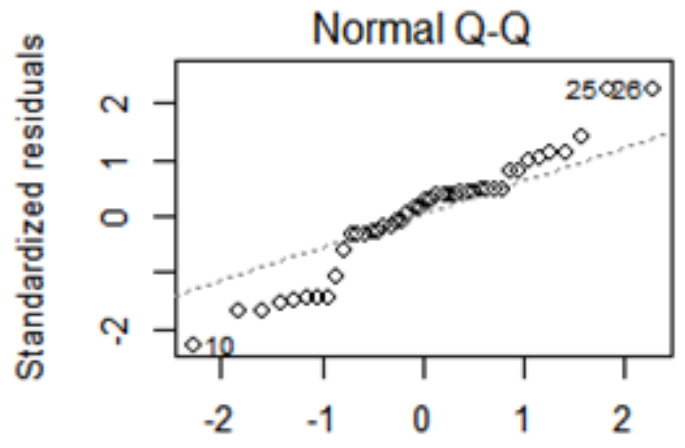

Theoretical Quantiles

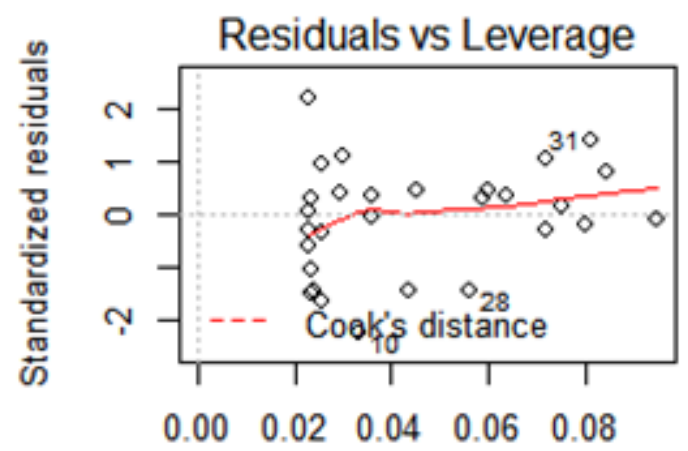

Leverage

Figure2. Scatter plot of Linear Regression models (Removed outliers). $y=0.9135 x+99.52, R^{2}=0.7091$

Step 1: The medical data imported from .xlsx file and attach() in R Studio and assign SBP as a dependent variable and Age as a independent variable, ie. $\left(X_{i}, Y_{i}\right) i=1,2, \ldots, n$

Step 2: To get summary statistics from the given data.

Step 3: To execute nonlinear regression function with $\mathrm{R}$ syntax and summarize the results

Step 4: Predict the given data and get Age2 predicted values.

Step 5. To get the residuals values for given data

Step 6: To get the standardized values for given data.

Step 7. To execute scale function of linear regression for the variable Age2 and get Standardized values for given data. Scale function of each element of those values is obtained by subtracting and dividing by standard deviation

Step 8. Repeat step 7 for SBP and get the standardized values for the given data

Step 9: To Visualize the results for nonlinear regression with help of different scatter plot.

The above algorithm execute the results of nonlinear regression of Predicted value, Standardized value, Residuals, Scale function value of Age, Age^2 and SBP values. (In Table 7 and 8, Figure 3). The results table and figure show the extremes in the $14^{\text {th }}$ row of the table are highlighted as outlier. In Visualization parts, models are fitted and highlighted in the scatter plots: Residuals vs, Fitted, Normal QQ plot, Scale - location and Residuals vs. Leverage. All the four plots show the 14 person as an outlier.

The summary statistics shows that the minimum age is 17 and maximum age is 69 , the minimum SBP is 110 and maximum SBP as 260 . The fitted linear regression model is $y=8 \mathrm{E}-04 \mathrm{x}^{2}+1.0015 \mathrm{x}+99.10$, $\mathrm{R}^{2}=0.4899$. In this model $\mathrm{R}^{2}$ value is less than 0.5 and this model is average. Subsequently the nonlinear regression function executes the above algorithm step 1 to step 9. Cook's distance is used in 
linear regression analysis to find influential outliers in a set of predictor variable. It is a way to identify points that negatively affects the regression model. The plot is a combination of each given data leverage and residual values, the higher leverage and residuals, the higher the Cook's. The F and t statistic are highly significant between two variables.

Table7. Descriptive Statistics $(n=45)$

\begin{tabular}{|l|l|}
\hline Regression Statistics \\
\hline R Square & 0.4899 \\
\hline Multiple R & 0.4656 \\
\hline Intercept & 99.104 \\
\hline Slope (Age) & 1.0015 \\
\hline Age $^{2}$ & -0.0008 \\
\hline
\end{tabular}

\section{R Studio Nonlinear Regression output:}

Residuals:

Min 1Q Median 3Q Max

$\begin{array}{lllll}-22.228 & -5.171 & 0.599 & 2.527 & 75.599\end{array}$

Coefficients:

Estimate Std. Error t value $\operatorname{Pr}(>|t|)$

(Intercept) $99.104712017 .03709725 .8177 .28 \mathrm{e}-07 * * *$

$\begin{array}{lllll}\text { Age } & 1.0015640 & 0.8473475 & 1.182 & 0.244\end{array}$

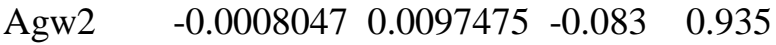

$---$

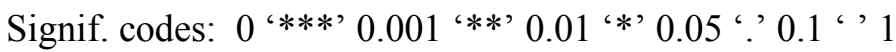

Residual standard error: 14.99 on 42 degrees of freedom

Multiple R-squared: 0.4899， Adjusted R-squared: 0.4656

F-statistic: 20.17 on 2 and 42 DF, p-value: $7.254 \mathrm{e}-07$

Table9. Nonlinear Regression Summary Output for Original Data

\begin{tabular}{|c|c|c|c|c|c|c|c|c|}
\hline $\begin{array}{c}\text { S. } \\
\text { No }\end{array}$ & $\begin{array}{c}\text { Age } \\
\left(x_{i}\right)\end{array}$ & $\begin{array}{c}\text { SBP } \\
\left(y_{i}\right)\end{array}$ & Age2 & $\begin{array}{c}\text { Predicted } \\
\text { Value } \\
\text { SBP }\end{array}$ & Standardized & Residuals & $\begin{array}{c}\text { Age2 } \\
\text { Standardized } \\
\left(x_{i}\right)\end{array}$ & $\begin{array}{c}\text { SBP } \\
\text { Standardized } \\
\left(y_{i}\right)\end{array}$ \\
\hline 1 & 65 & 162 & 4225 & 160.807 & 0.08349 & 1.19329 & 1.50999 & 0.98387 \\
\hline 2 & 46 & 142 & 2116 & 143.474 & -0.1005 & -1.474 & -0.0665 & 0.00867 \\
\hline 3 & 67 & 170 & 4489 & 162.597 & 0.52733 & 7.40259 & 1.70733 & 1.37395 \\
\hline 4 & 42 & 124 & 1764 & 139.751 & -1.0756 & -15.751 & -0.3296 & -0.869 \\
\hline 5 & 67 & 158 & 4489 & 162.597 & -0.3275 & -4.5974 & 1.70733 & 0.78883 \\
\hline 6 & 56 & 154 & 3136 & 152.669 & 0.09046 & 1.3311 & 0.69598 & 0.59379 \\
\hline 7 & 64 & 162 & 4096 & 159.909 & 0.1453 & 2.09105 & 1.41356 & 0.98387 \\
\hline 8 & 56 & 150 & 3136 & 152.669 & -0.1814 & -2.6689 & 0.69598 & 0.39875 \\
\hline 9 & 59 & 140 & 3481 & 155.396 & -1.0497 & -15.396 & 0.95386 & -0.0889 \\
\hline 10 & 34 & 110 & 1156 & 132.228 & -1.5156 & -22.228 & -0.7841 & -1.5517 \\
\hline 11 & 42 & 128 & 1764 & 139.751 & -0.8024 & -11.751 & -0.3296 & -0.674 \\
\hline 12 & 48 & 130 & 2304 & 145.326 & -1.0442 & -15.326 & 0.07407 & -0.5765 \\
\hline 13 & 39 & 144 & 1521 & 136.942 & 0.48187 & 7.05817 & -0.5112 & 0.10619 \\
\hline 14 & 47 & 220 & 2209 & 144.401 & 5.15383 & 75.5993 & 0.00306 & 3.81195 \\
\hline 15 & 45 & 138 & 2025 & 142.546 & -0.3102 & -4.5457 & -0.1345 & -0.1864 \\
\hline 16 & 47 & 145 & 2209 & 144.401 & 0.04085 & 0.59926 & 0.00306 & 0.15495 \\
\hline 17 & 65 & 162 & 4225 & 160.807 & 0.08349 & 1.19329 & 1.50999 & 0.98387 \\
\hline 18 & 45 & 135 & 2025 & 142.546 & -0.5149 & -7.5457 & -0.1345 & -0.3327 \\
\hline 19 & 17 & 114 & 289 & 115.899 & -0.1393 & -1.8988 & -1.4321 & -1.3566 \\
\hline 20 & 20 & 116 & 400 & 118.814 & -0.1994 & -2.8141 & -1.3492 & -1.2591 \\
\hline
\end{tabular}


Detecting Outliers using R Package in Fitting Data with Linear and Nonlinear Regression Models

\begin{tabular}{|c|c|c|c|c|c|c|c|c|}
\hline 21 & 19 & 124 & 361 & 117.844 & 0.44048 & 6.15605 & -1.3783 & -0.869 \\
\hline 22 & 36 & 136 & 1296 & 134.118 & 0.12837 & 1.88181 & -0.6794 & -0.2839 \\
\hline 23 & 50 & 142 & 2500 & 147.171 & -0.3519 & -5.1713 & 0.22058 & 0.00867 \\
\hline 24 & 39 & 120 & 1521 & 136.942 & -1.1566 & -16.942 & -0.5112 & -1.0641 \\
\hline 25 & 21 & 120 & 441 & 119.783 & 0.01527 & 0.2173 & -1.3185 & -1.0641 \\
\hline 26 & 44 & 160 & 1936 & 141.616 & 1.25492 & 18.3843 & -0.201 & 0.88635 \\
\hline 27 & 44 & 160 & 1936 & 141.616 & 1.25492 & 18.3843 & -0.201 & 0.88635 \\
\hline 28 & 53 & 158 & 2809 & 149.927 & 0.54855 & 8.07267 & 0.45155 & 0.78883 \\
\hline 29 & 63 & 144 & 3969 & 159.01 & -1.0371 & -15.01 & 1.31863 & 0.10619 \\
\hline 30 & 29 & 130 & 841 & 127.473 & 0.17253 & 2.52665 & -1.0195 & -0.5765 \\
\hline 31 & 25 & 125 & 625 & 123.641 & 0.09357 & 1.3591 & -1.181 & -0.8203 \\
\hline 32 & 69 & 175 & 4761 & 164.382 & 0.77579 & 10.6183 & 1.91064 & 1.61775 \\
\hline 33 & 56 & 154 & 3136 & 152.669 & 0.09046 & 1.3311 & 0.69598 & 0.59379 \\
\hline 34 & 64 & 162 & 4096 & 159.909 & 0.1453 & 2.09105 & 1.41356 & 0.98387 \\
\hline 35 & 36 & 136 & 1296 & 134.118 & 0.12837 & 1.88181 & -0.6794 & -0.2839 \\
\hline 36 & 50 & 142 & 2500 & 147.171 & -0.3519 & -5.1713 & 0.22058 & 0.00867 \\
\hline 37 & 39 & 120 & 1521 & 136.942 & -1.1566 & -16.942 & -0.5112 & -1.0641 \\
\hline 38 & 21 & 120 & 441 & 119.783 & 0.01527 & 0.2173 & -1.3185 & -1.0641 \\
\hline 39 & 53 & 158 & 2809 & 149.927 & 0.54855 & 8.07267 & 0.45155 & 0.78883 \\
\hline 40 & 63 & 144 & 3969 & 159.01 & -1.0371 & -15.01 & 1.31863 & 0.10619 \\
\hline 41 & 29 & 130 & 841 & 127.473 & 0.17253 & 2.52665 & -1.0195 & -0.5765 \\
\hline 42 & 20 & 116 & 400 & 118.814 & -0.1994 & -2.8141 & -1.3492 & -1.2591 \\
\hline 43 & 19 & 124 & 361 & 117.844 & 0.44048 & 6.15605 & -1.3783 & -0.869 \\
\hline 44 & 36 & 136 & 1296 & 134.118 & 0.12837 & 1.88181 & -0.6794 & -0.2839 \\
\hline 45 & 50 & 142 & 2500 & 147.171 & -0.3519 & -5.1713 & 0.22058 & 0.00867 \\
\hline
\end{tabular}

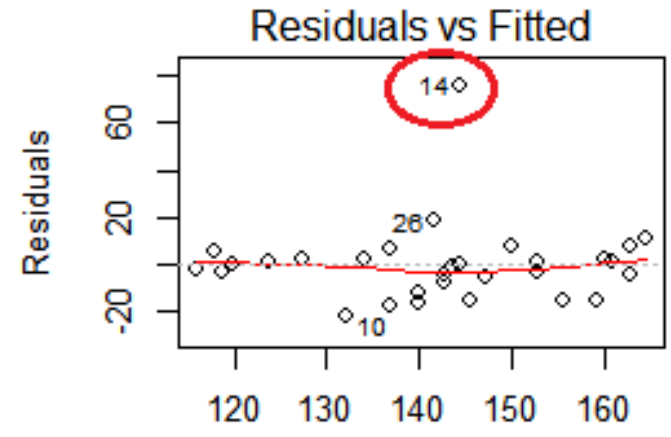

Fitted values

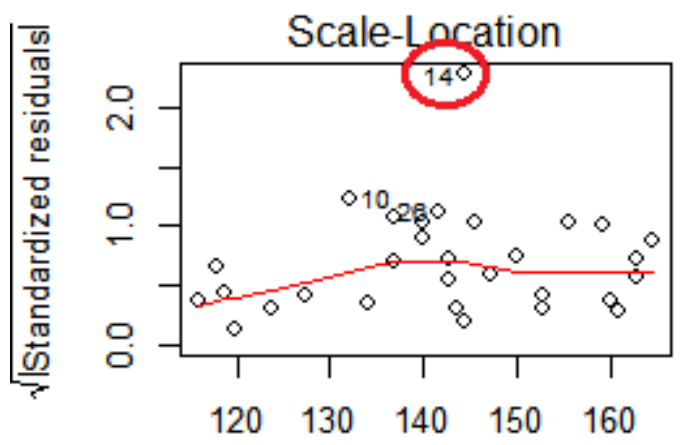

Fitted values

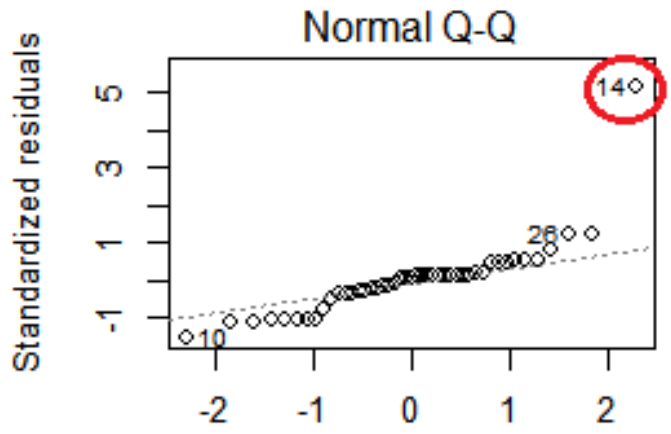

Theoretical Quantiles

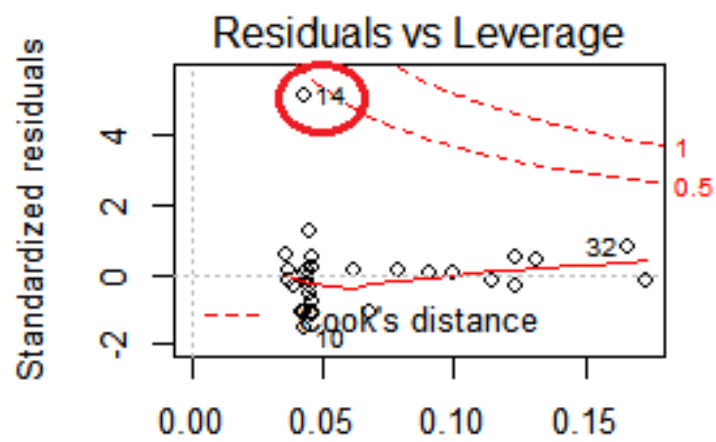

Leverage

Figure3. Scatter plot for Nonlinear Regression (with outliers) $Y=8 E-04 x^{2}+1.0015 x+99.10, R^{2}=0.4899$ 
The previous algorithm again execute the results of linear regression for outlier data and get the Predicted value, Standardized value, Residuals, Scale function value of Age and SBP values. (Table 10 to 11, Figure 4). The linear regression model improves better. In Visualization parts, models are fitted and highlighted in the plots: Residuals vs, Fitted, Normal QQ plot, Scale - location and Residuals vs. Leverage. All the four plots show better results after the removal of outliers.

The summary statistics shows that the minimum age is 17 and maximum age is 69 , the minimum SBP is 110 and maximum SBP as 175 . The fitted linear regression model is $y=0.0098 x^{2}-0.0363+0.109$, $\mathrm{R}^{2}=0.7169$. In this model $\mathrm{R}^{2}$ value is closer to .705 and this model is better. Subsequently the non linear regression executes the algorithm step 1 to step 9. Cook's distance is used in linear regression analysis to find influential outliers in a set of predictor variable. It is a way to identify points that negatively affects the regression model. The plot is a combination of each given data leverage and residual values, the higher leverage and residuals, the higher the Cook's. The F and t statistic are highly significant between two variables.

Table10. Descriptive Statistics $n=44$

\begin{tabular}{|l|l|}
\hline Regression Statistics & \\
\hline R Square & 0.7169 \\
\hline Multiple R & 0.7031 \\
\hline Intercept & 0.0109 \\
\hline Age $^{2}$ & 0.0105 \\
\hline
\end{tabular}

R Studio Nonlinear Regression output (Outlier Removed):

Residuals:

Min 1Q Median 3Q Max

$\begin{array}{lllll}-18.9926 & -5.2332 & 0.6652 & 4.4728 & 22.7874\end{array}$

Coefficients:

Estimate Std. Error $t$ value $\operatorname{Pr}(>|t|)$

(Intercept) $116.81030 \quad 2.65684 \quad 43.97<2 \mathrm{e}-16 * * *$

Age2 $\quad 0.01054 \quad 0.00103 \quad 10.235 .68 \mathrm{e}-13 * * *$

$---$

Signif. codes: 0 '***’ 0.001 '**’ 0.01 '*’ 0.05 '?' 0.1 ' ' 1

Residual standard error: 9.142 on 42 degrees of freedom

Multiple R-squared: 0.7136, Adjusted R-squared: 0.7068

F-statistic: 104.6 on 1 and 42 DF, p-value: 5.676e-13

Table11. After removing outlier from original data (removed $14^{\text {th }}$ row)

\begin{tabular}{|c|c|c|c|c|c|c|c|c|}
\hline $\begin{array}{c}\text { S. } \\
\text { No }\end{array}$ & $\begin{array}{c}\text { Age } \\
\left(x_{i}\right)\end{array}$ & $\begin{array}{c}\text { SBP } \\
\left(y_{i}\right)\end{array}$ & Age2 & $\begin{array}{c}\text { Predicted } \\
\text { Value }\end{array}$ & Standardized & Residuals & $\begin{array}{c}\text { Age2 } \\
\text { Standardized } \\
\left(x_{i}\right)\end{array}$ & $\begin{array}{c}\text { SBP } \\
\text { Standardized } \\
\left(y_{i}\right)\end{array}$ \\
\hline 1 & 65 & 162 & 4225 & 161.335 & 0.07563 & 0.66516 & 1.32603 & 1.30043 \\
\hline 2 & 46 & 142 & 2116 & 139.11 & 0.31985 & 2.89054 & 0.10515 & 0.11577 \\
\hline 3 & 67 & 170 & 4489 & 164.117 & 0.67421 & 5.88303 & 1.45455 & 1.77429 \\
\hline 4 & 42 & 124 & 1764 & 135.4 & -1.263 & -11.4 & -0.1519 & -0.9504 \\
\hline 5 & 67 & 158 & 4489 & 164.117 & -0.701 & -6.117 & 1.45455 & 1.0635 \\
\hline 6 & 56 & 154 & 3136 & 149.859 & 0.46085 & 4.14142 & 0.74772 & 0.82657 \\
\hline 7 & 64 & 162 & 4096 & 159.975 & 0.22941 & 2.02461 & 1.26178 & 1.30043 \\
\hline 8 & 56 & 150 & 3136 & 149.859 & 0.01574 & 0.14142 & 0.74772 & 0.58964 \\
\hline 9 & 59 & 140 & 3481 & 153.494 & -1.5092 & -13.494 & 0.94049 & -0.0027 \\
\hline 10 & 34 & 110 & 1156 & 128.993 & -2.1167 & -18.993 & -0.6659 & -1.7797 \\
\hline 11 & 42 & 128 & 1764 & 135.4 & -0.8198 & -7.4 & -0.1519 & -0.7135 \\
\hline
\end{tabular}


Detecting Outliers using R Package in Fitting Data with Linear and Nonlinear Regression Models

\begin{tabular}{|c|c|c|c|c|c|c|c|c|}
\hline 12 & 48 & 130 & 2304 & 141.091 & -1.2273 & -11.091 & 0.23366 & -0.595 \\
\hline 13 & 39 & 144 & 1521 & 132.839 & 1.2387 & 11.1609 & -0.3447 & 0.23424 \\
\hline 15 & 45 & 138 & 2025 & 138.151 & -0.0167 & -0.1505 & 0.04089 & -0.1212 \\
\hline 16 & 47 & 145 & 2209 & 140.09 & 0.54334 & 4.91048 & 0.16941 & 0.29347 \\
\hline 17 & 65 & 162 & 4225 & 161.335 & 0.07563 & 0.66516 & 1.32603 & 1.30043 \\
\hline 18 & 45 & 135 & 2025 & 138.151 & -0.3487 & -3.1505 & 0.04089 & -0.2989 \\
\hline 19 & 17 & 114 & 289 & 119.856 & -0.664 & -5.8559 & -1.7583 & -1.5427 \\
\hline 20 & 20 & 116 & 400 & 121.026 & -0.5682 & -5.0257 & -1.5655 & -1.4243 \\
\hline 21 & 19 & 124 & 361 & 120.615 & 0.38314 & 3.38535 & -1.6298 & -0.9504 \\
\hline 22 & 36 & 136 & 1296 & 130.468 & 0.61542 & 5.53199 & -0.5374 & -0.2396 \\
\hline 23 & 50 & 142 & 2500 & 143.156 & -0.128 & -1.1562 & 0.36218 & 0.11577 \\
\hline 24 & 39 & 120 & 1521 & 132.839 & -1.425 & -12.839 & -0.3447 & -1.1874 \\
\hline 25 & 21 & 120 & 441 & 121.458 & -0.1647 & -1.4577 & -1.5013 & -1.1874 \\
\hline 26 & 44 & 160 & 1936 & 137.213 & 2.52259 & 22.7875 & -0.0234 & 1.18196 \\
\hline 27 & 44 & 160 & 1936 & 137.213 & 2.52259 & 22.7875 & -0.0234 & 1.18196 \\
\hline 28 & 53 & 158 & 2809 & 146.413 & 1.28519 & 11.5875 & 0.55495 & 1.0635 \\
\hline 29 & 63 & 144 & 3969 & 158.637 & -1.6533 & -14.637 & 1.19752 & 0.23424 \\
\hline 30 & 29 & 130 & 841 & 125.673 & 0.48466 & 4.32694 & -0.9872 & -0.595 \\
\hline 31 & 25 & 125 & 625 & 123.397 & 0.18034 & 1.60323 & -1.2443 & -0.8912 \\
\hline 32 & 69 & 175 & 4761 & 166.983 & 0.92726 & 8.0166 & 1.58306 & 2.07045 \\
\hline 33 & 56 & 154 & 3136 & 149.859 & 0.46085 & 4.14142 & 0.74772 & 0.82657 \\
\hline 34 & 64 & 162 & 4096 & 159.975 & 0.22941 & 2.02461 & 1.26178 & 1.30043 \\
\hline 35 & 36 & 136 & 1296 & 130.468 & 0.61542 & 5.53199 & -0.5374 & -0.2396 \\
\hline 36 & 50 & 142 & 2500 & 143.156 & -0.128 & -1.1562 & 0.36218 & 0.11577 \\
\hline 37 & 39 & 120 & 1521 & 132.839 & -1.425 & -12.839 & -0.3447 & -1.1874 \\
\hline 38 & 21 & 120 & 441 & 121.458 & -0.1647 & -1.4577 & -1.5013 & -1.1874 \\
\hline 39 & 53 & 158 & 2809 & 146.413 & 1.28519 & 11.5875 & 0.55495 & 1.0635 \\
\hline 40 & 63 & 144 & 3969 & 158.637 & -1.6533 & -14.637 & 1.19752 & 0.23424 \\
\hline 41 & 29 & 130 & 841 & 125.673 & 0.48466 & 4.32694 & -0.9872 & -0.595 \\
\hline 42 & 20 & 116 & 400 & 121.026 & -0.5682 & -5.0257 & -1.5655 & -1.4243 \\
\hline 43 & 19 & 124 & 361 & 120.615 & 0.38314 & 3.38535 & -1.6298 & -0.9504 \\
\hline 44 & 36 & 136 & 1296 & 130.468 & 0.61542 & 5.53199 & -0.5374 & -0.2396 \\
\hline 45 & 50 & 142 & 2500 & 143.156 & -0.128 & -1.1562 & 0.36218 & 0.11577 \\
\hline
\end{tabular}

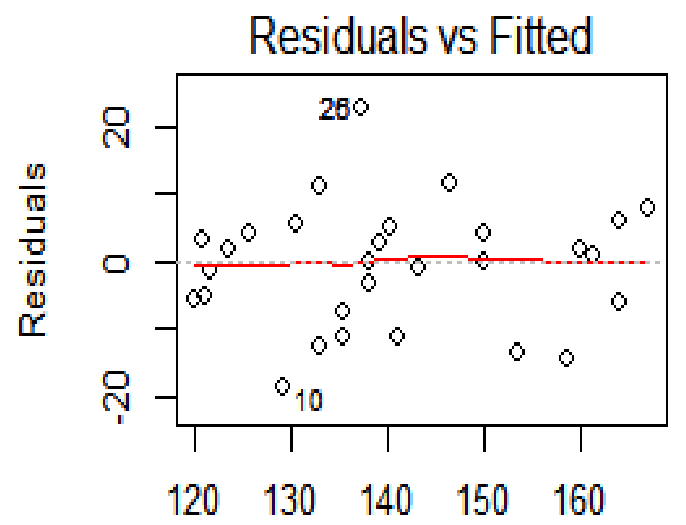

Fitted values

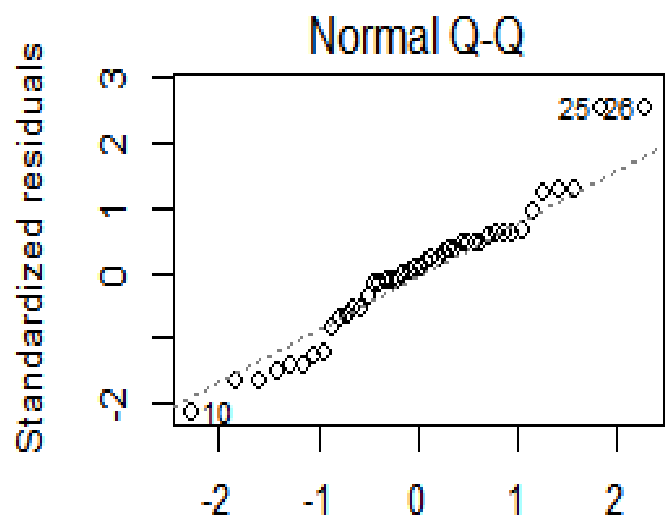

Theoretical Quantiles 


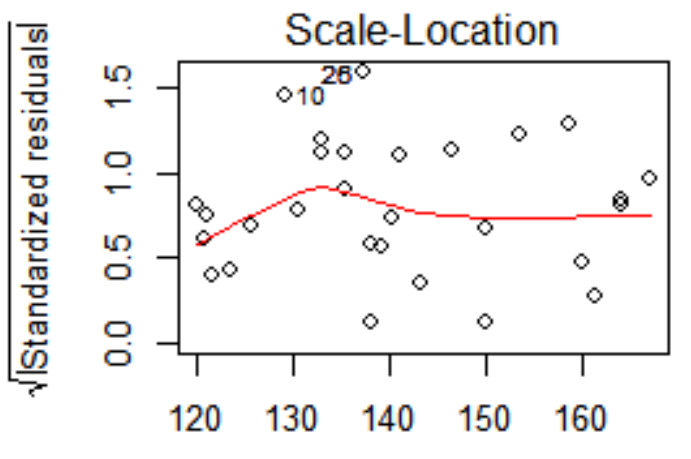

Fitted values

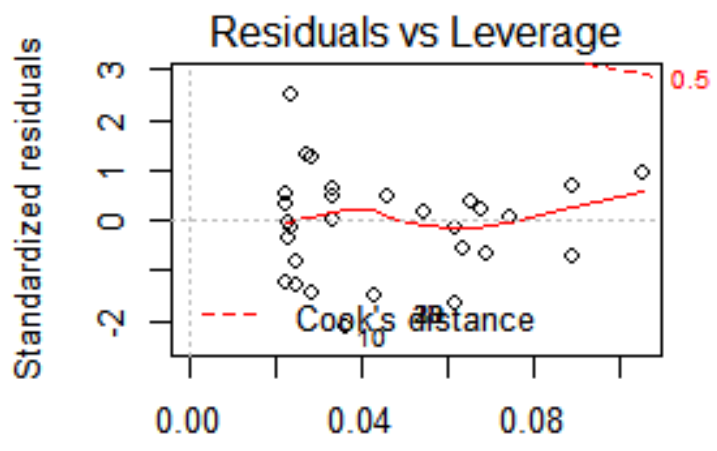

Leverage

Figure4. Scatter plot for Nonlinear Regression (Outlier Removed) $y=-2 E-06 x^{2}+0.0183 x+112.2, R^{2}=0.4904$

\section{CONClusion}

Linear and non linear regression analysis assumes scatter of data, fitting of straight line or normal distribution. An outlier is an extreme observation when the residual is larger in absolute value when compared with the other observed data set. The detection of outlier can be defined as the process of detecting and subsequently excluding outliers from the given set of data. Outlier can dominate the sum of the square calculation and might lead to misleading results.

The linear and nonlinear regression model fitted for original and outlier removed data. The results of original data linear and non linear regression are not a good model. In both model $\mathrm{R}^{2}$ value is less than 0.5 . After removal of the outlier, it achieved better fit of linear and nonlinear regression model. The $\mathrm{R}^{2}$ values are more than 0.7 . The $\mathrm{F}$ and $\mathrm{t}$ statistic are significant in two models. The scatter plot clearly visualizes the outlier and without outlier data for different plots. In this paper, the detection of outliers in simple linear regression model has been discussed. A new approach for detecting outliers without the use of predicted values have been proposed, which is quite useful in detecting outliers that detects the outliers as same as the residual and standardized residual method. Hence, it is proposed, that in simple linear regression model, the difference method can be used for detecting outliers. In general the linear and nonlinear regression model is used to removal of outlier for any given data set.

\section{REFERENCES}

[1] N. R. Draper, Norman Richard Draper, Harry Smith (1981), Applied Regression Analysis, Third Edition, A John Wiley \& Sons, Inc., Publication, New Jersey, USA.

[2] Framstad, Erik, Steinar Engen, and Nils Chr. "Regression analysis, residual analysis and missing variables in regression models." Oikos (1985): 319-323.

[3] Manimannan G. and R. Lakshmi Priya (2019), "Evaluation and Classification of Master Health Checkup Database using Data mining Techniques", International Journal of Data Mining and Emerging Technologies, Vol.9, Isssue:2 pp. 25-32.

[4] Douglas Montgomery, et. al. (2012) Introduction to Linear Regression Analysis, Fifth Edition, A John Wiley \& Sons, Inc., Publication, New Jersey, USA.

[5] Bipin Gogoi and Mintu Kr. Das. Usage of graphical displays to detect outlying observations in linear regression. Indian Journal of Applied Research 5.5 (2015): 19-24.

[6] Hampel, F.R. et.al (1986), Robust Statistics: The Approach Based on Influence Functions. New York: John Wiley \& Sons, Inc.

Citation: Manimannan G,et.al., Detecting Outliers using R Package in Fitting Data with Linear and Nonlinear Regression Models, International Journal of Scientific and Innovative Mathematical Research (IJSIMR), vol. 8, no. 4, pp. 1-13, 2020. Available : DOI: https://doi.org/10.20431/2347-3142.0804001

Copyright: () 2020 Authors. This is an open-access article distributed under the terms of the Creative Commons Attribution License, which permits unrestricted use, distribution, and reproduction in any medium, provided the original author and source are credited. 